\title{
Morphological Factors and Cardiac Doses in Whole Breast Radiation for Left-sided Breast Cancer
}

\author{
Hui Guan', Yuan-Li Dong', Li-Jie Ding ${ }^{3}$, Zi-Cheng Zhang², Wei Huang ${ }^{2}$, Cheng- \\ Xin Liu', ${ }^{1,2}$ Cheng-Rui Fü, ${ }^{1,2}$ Jian $\mathrm{Zhu}^{2}$, Hong-Sheng Li², Miao-Miao $\mathrm{Li}^{4}$, Bao- \\ Sheng $\mathrm{Li}^{2 *}$
}

\begin{abstract}
Background: To investigate the impact of the breast size, shape, maximum heart depth (MDH), and chest wall hypotenuse (the distance connecting middle point of the sternum and the length of lung draw on the selected transverse CT slice) on the volumetric dose to heart with whole breast irradiation (WBI) of left-sided breast cancer patients. Materials and Methods: Fifty-three patients with left-sided breast cancer undergoing adjuvant intensity-modulated radiotherapy (IMRT) were enrolled in the study. The primary breast size and shape, MHD and $D_{\mathrm{CWH}}$ (chest wall hypotenuse) were contoured on radiotherapy $(\mathrm{RT})$ planning $\mathrm{CT}$ slices. The dose data of hearts were obtained from the dose-volume histograms (DVHs). Data were analyzed by one-way analysis of variance (ANOVA), Student's t-test and linear regression analysis. Results: Breast size was independent of heart dose, whereas breast shape, MHD and DCWH were correlated with heart dose. The shapes of breasts were divided into four types, as the flap type, hemisphere type, cone type and pendulous type with heart mean dose being 491.8 $\pm 234.6 \mathrm{cGy}, 752.7 \pm 219.0 \mathrm{cGy}, 620.2 \pm 275.7 \mathrm{cGy}$, and $666.1 \pm 238.0 \mathrm{cGy}$, respectively. The flap type of breasts shows a strong statistically reduction in heart dose, compared to others ( $p=0.008$ for V30 of heart). DCWH and MHD were found to be the most important parameters correlating with heart dose in WBI. Conclusions: More attention should be paid to the heart dose of non-flap type patients. The MHD was found to be the most important parameter to correlate with heart dose in tangential WBI, closely followed by the DCWH, which could help radiation oncologists and physicsts evaluate heart dose and design RT plan in advance.
\end{abstract}

Keywords: Breast cancer - intensity-modulated radiotherapy - breast size - maximum heart distance

Asian Pac J Cancer Prev, 16 (7), 2889-2894

\section{Introduction}

Breast cancer is one of the most common neoplasms affecting women. Postoperative radiotherapy (RT) after lumpectomy provides a substantial reduction in local and regional recurrence rates for early-stage and locally advanced breast cancers and contributes to improvements in overall survival (Clarke et al., 2005). Although studies have consistently shown the reduction of local regional recurrence and cause specific death, many of the earlier studies did not show an increase in overall survival for breast patients treated with RT largely because of the increase in non-breast cancer mortality, particularly vascular/cardiac mortality (Rutqvist and Johansson, 1990; Group, 2000). Rates of major coronary events increased linearly with the mean dose to the heart by $7.4 \%$ per gray (95\% confidence interval, 2.9 to $14.5 ; p<0.001$ ), with no apparent threshold (Darby et al., 2013). The cardiac complications induced by whole breast irradiation (WBI) have been shown to be directly related to the irradiated volume, dose, and treatment technique (Das et al., 1998). Modern treatment techniques with CT-based planning have allowed better selection and placement of beam angle and energy with nondivergent and coplanar beams to spare organs at risks (OARs). Henceforce, it is of importance for cardiac dose to be assessed in early stage breast cancer patients received intensity-modulated radiotherapy (IMRT) (Mansouri et al., 2014). Most previous research only demonstrated bigger breasts increased cardiac dose, but no relationship to the shape of breasts and thoracic cage were reported (Neal et al., 1995; Bhatnagar et al., 2006; Hannan et al., 2012).

Therefore, the objective of this retrospective study was to determine the impact of breast size and shape, MHD and DCWH on heart dose in WBI of left-sided breast patients.

\section{Materials and Methods}

\section{Characteristics of patients and breasts}

Between November 2010 and December 2013, fifty-

${ }^{I}$ Department of Radiation Oncology, Shandong's Key Laboratory of Radiation Oncology, Shandong Cancer Hospital, School of Medicine and Life Sciences, University of Jinan and Shandong Academy of Medical Sciences, ${ }^{2}$ Department of Oncology, Shandong Cancer Hospital, ${ }^{3}$ Department of Epidemiology and Biostatistics, School of Public Health, Shandong University, ${ }^{4}$ Shandong Medical College, Jinan, Shandong, China*For correspondence: baoshli@yahoo.com 


\section{Hui Guan et al}

three consecutive patients with left-sided breast cancer after breast-conserving operation in Shandong Cancer Hospital \& Institute were enrolled to this retrospective study. This observational study has been approved by the Institutional Review Board of Shandong Cancer Hospital and Institute and informed consent of retaining data for subsequent research from each patient was obtained. In this study, breast characteristics were separated into two components: size and shape. Breast volume, measured in cubic centimeters (cc) from PTV, was used as a surrogate for breast size which has been validated as effective in previous studies (Neal et al., 1995; Dundas et al., 2007; Hannan et al., 2012).

Consecutive WBI patients were included in 1 of 3 cohorts according to breast size: large breast (PTV $>1200$ cc) $(8$ pts $)$, medium breast $(1200 \mathrm{cc}>\mathrm{PTV}>800 \mathrm{cc})(21$ pts) or small breast (PTV $<800 \mathrm{cc})(24 \mathrm{pts})$. Breast shape, according to the protruding length of breasts, can be divided into the following types (Figure 1): $i$ ) Flat type: the protruding length is less than the radius of breasts base. Breasts shaped like a disk elevate gradually over the chest wall with unclear boundary. And there is no difference between the standing and supine position. ii) Hemisphere type: the protruding length is equal to the radius of the base of breasts. Breasts shaped like hemisphere elevate sharply over the chest wall with clear boundary. The curve of breasts still can be seen in supine position. iii) Cone type: the protruding length is greater than the radius of the base of breasts. The breast angle formed by the lower edge of the chest wall is less than $90^{\circ}$. The arc formed in upright position can easily been seen. (iv) Pendulous shape: the protruding length of breasts is even greater, shaped like pendulous bags in standing position and extending outwards in supine position.

\section{Definitions of morphological parameters}

According to methods established by Qi et al. (2012), one transverse CT slice was selected for the delineation of all the parameters. The selected plane was defined as the top slice of the right side of the diaphragm. One line (AB) connecting middle point of the front edge of sternum (A) and the end of both lungs (B) was drawn. The second

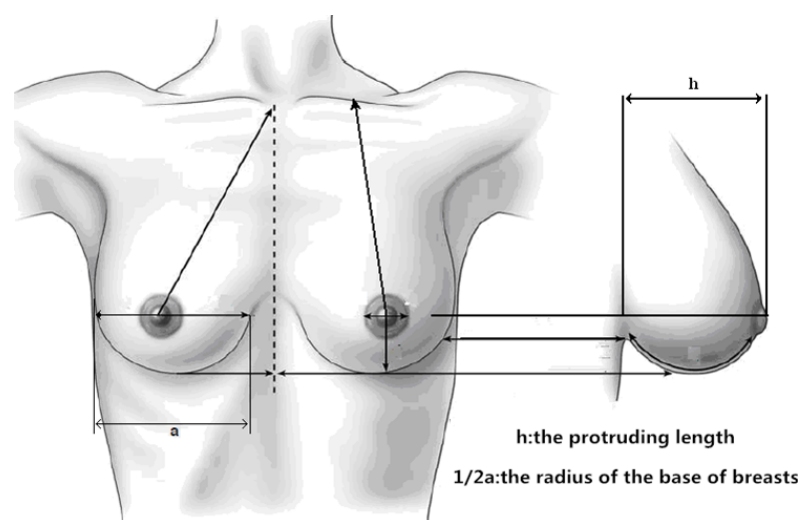

Figure 1. The Measurement of the Breast Shape Sketch, Including the Standard Parameter of Height of Nipple, Base Width, Internipple Distance, as Well as The Sternal Notch to the Nipple Distance, the Protruding Length (h) and the Radius of the Base of Breasts (1/2a) line $(\mathrm{CM})$ was the perpendicular bisector of Line $\mathrm{AB}$. As shown in Figure 1, DCWH, the distance between from the point $\mathrm{A}$ and point $\mathrm{M}$, represents chest wall hypotenuse ; MHD is the distance of the forefront of the heart to the Line AM (Qi et al., 2012).

\section{$R T$ and dose calculation}

Each patient positioned supine with their arms raised above their heads as reported previously (Qi et al., 2010; Ding et al., 2014). Radiopaque wire was used to delineate medial, inferior, lateral, and superior field borders (mid sternum, $2 \mathrm{~cm}$ inferior to infra-mammary line, mid posterior axillary line, and $2 \mathrm{~cm}$ superior to palpable breast tissue, respectively). The surgical scar and contralateral breast were also delineated with wire when applicable. Tangential fields were established by use of the Source Axis Distance technique (isocenter within breast) for supine patients. Tangential field borders were initially aligned to anatomic guidewires and subsequently finalized per CT scan to ensure coverage of the entire breast with a posterior match line.

A planning CT scan at a slice thickness of $3 \mathrm{~mm}$ was performed and transferred to an Varian Eclipse RT treatment planning system (TPS, version 8.6, Varian Medical Systems, Palo Alto, CA, USA) for delineation and planning. The whole breast reference volume was defined per Radiation Therapy Oncology Group (RTOG) guidelines as all tissues excluding lung and intrathoracic/ intra-abdominal structures located within the tangential fields (RTOG 0413). For this study, a standardized PTV was established for all patients. The guideline for the posterior edge of the PTV was that it had the same angle throughout the entire CT dataset per patient. This edge typically ran from the patients' midlines medially to their mid-axillary lines posteriolaterally. These borders were determined by the radiation oncologist and drawn on all CT scans superiorly up to $10 \mathrm{~mm}$ within the superior field edge and inferiorly to infra-mammary fold. The posterior edge was clipped at the lung interface, and the anterior and lateral borders were $5 \mathrm{~mm}$ inside the patients' external skin contour. These borders were chosen to eliminate penumbra, skin and non-breast tissue from the PTV, although still reflecting the irradiated volume of the technique. The standardized PTV was used as a variable

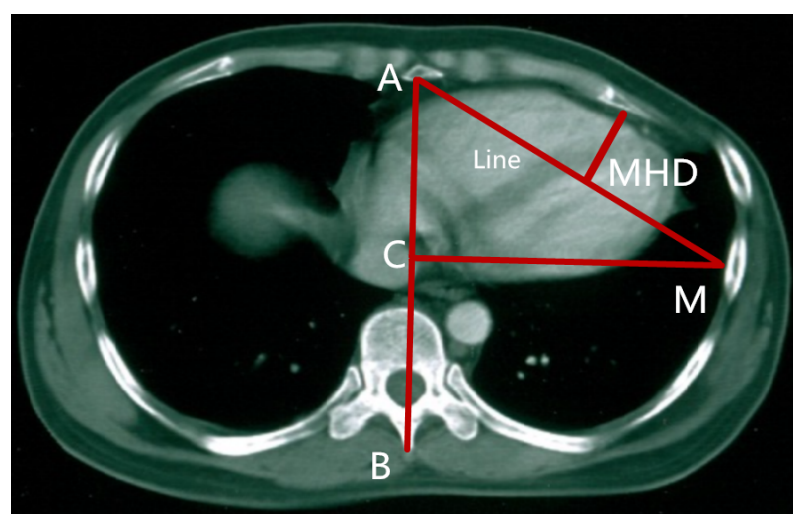

Figure 2. Definitions of MHD (Maximum Heart Depth) and DCWH (the Line Which Connect the Middle Point of the Front Edge of Sternum (A) and the Middle of Both Lungs (M)) 
across all participants.

The primary breast volume was calculated by TPS. The mean heart doses were obtained from the dose-volume histogram (DVH) of the IMRT plan and also reported as a percentage of the prescribed dose to the whole breast. The presence of any portion of the heart within the treatment field was recorded and DCWH, MHD were measured for each patient, with the two observers (the same radiation physicist and radiation oncologist) reaching a consensus for each measurement.

The indices of MHD and DCWH were used to correlate with the dosimetric data. According to the shape of target region, two parallel opposed tangential fields were designed. To avoid radiation omission in the target region of the lacteal gland caused by respiratory movement, the front limit of the fields was set at $20 \mathrm{~mm}$ off the skin surface (Vicini et al., 2002). Three or four apertures on each direction of the tangential field were designed to shield the lung and reduce the high-dose region in the target region. Dose rate was set at $300 \mathrm{MU} /$ min. Then distribution of dose curves was regulated precisely through adjusting the weight between extended fields and apertures as well as by the shape of MLC (Yin et al., 2012).

The prescription dose was 50.4 Gy (1.8 Gy/fraction) to the whole breast and followed by a photon or electron boost dose of 10 to $12 \mathrm{~Gy}$ to lumpectomy cavity. A plan was considered acceptable if at least $95 \%$ of PTV received the prescription dose and when at least $90 \%$ of the target breast received $95 \%$ of prescription. All plans met Quantitative Analysis of Normal Tissue Effects in the Clinic (QUANTEC) recommended dose constraints. Patient setup position was verified before treatment per week by kilo-voltage cone beam CT (CBCT).

\section{Statistical analysis}

Analyses were performed using the Statistical Package for Social Sciences, version 16.0 (SPSS, Chicago, IL, USA). Quantitative data were as expressed as mean \pm standard deviation (SD). One-way analysis of variance (ANOVA) was used to determine significance in the difference for parameters of cardiac dose among four types of breast shape and breast size. Statistical analysis of two categories was performed with Student's t-test. A Pearson correlation analysis was performed for breast volume, heart, and contralateral breast doses. Linear regression analysis was used for correlations among heart mean dose, V25, MHD, and DCWH. All $p$ values were two-sided, and less than 0.05 was considered statistically significant.

\section{Results}

\section{Patient characteristics}

The characteristics of patients are summarized in Table 1. The median age was 43 years (range, $32-71$ years). The PTV (and SD) was 892.9 \pm 372.6 . We divided our patients into four groups according to the breast shape of aesthetics. The mean length of MHD (and SD) was $365 \pm 0.8$ (range, $0.1-3.9 \mathrm{~cm}$ ). The mean length of DCWH (and SD) was $1.4 \pm 0.9$ (range, $1.3-1.8 \mathrm{~cm}$ ).

Effect of breast morphological characters on dosimetry Relationship between breast size and heart dose

Dosimetry for the heart were unaffected by breast size ( $p=0.2$ for $\mathrm{V} 30$ of heart). Interestingly, the heart dose varied significantly within each cohort, the small-breasted cohort showed that heart dose was markedly decreased compared with the others in ANOVA, as Table 2 shows.

\section{Relationship between breast shape and heart dose}

Heart dose was markedly decreased in the flap type compared with the others (Table 3). However, heart dosimetric parameters were indistinguishable among the other three types (Table 4).

\section{Effect of MHD and DCWH on heart dose}

Not only DCWH but also MHD shows statistically correlationship with the cardiac dose. Linear regression analysis was then performed to correlate MHD, DCWH and heart dose. For left-sided patients, the mean dose of heart and percentage volume of heart at different dose levels correlated fairly with MHD/DCWH (cm) as the following relationships:

Dmean=1911.4-93.2·DCWH $(\mathrm{R} 2=0.168)$;

Dmean=192.2+215.7·MHD (R2=0.352);

Table 1. Main Patient Characteristics

\begin{tabular}{|c|c|c|}
\hline Characteristics & Parameters & Values \\
\hline \multicolumn{3}{|l|}{ Age (y) } \\
\hline & Mean & 43.3 \\
\hline & Range & $32-71$ \\
\hline \multicolumn{3}{|l|}{ KPS } \\
\hline & Median & 90 \\
\hline & Range & $80-100$ \\
\hline \multicolumn{3}{|c|}{ Body Mass Index } \\
\hline & Mean & 24.4 \\
\hline & $\mathrm{SD}$ & $17.9-35.3$ \\
\hline \multicolumn{3}{|c|}{ Pathological Tumor Stage } \\
\hline & Tis & 1 \\
\hline & $\mathrm{T} 1$ & 41 \\
\hline & $\mathrm{T} 2$ & 11 \\
\hline \multicolumn{3}{|c|}{ Pathological Nodal Stage } \\
\hline & No & 48 \\
\hline & N1 & 5 \\
\hline \multicolumn{3}{|c|}{ Estrogen/Progesteron Receptors } \\
\hline & Positive & 39 \\
\hline & Negative & 14 \\
\hline \multicolumn{3}{|c|}{ Anti-hormone Therapy } \\
\hline & Yes & 2 \\
\hline & No & 51 \\
\hline \multicolumn{3}{|c|}{ Adjuvant Chemotherapy } \\
\hline & Yes & 31 \\
\hline & No & 22 \\
\hline \multicolumn{3}{|l|}{ Breast Size } \\
\hline & Mean & 892.9 \\
\hline & $\mathrm{SD}$ & 372.5 \\
\hline & $>1200 \mathrm{~cm} 3$ & 8 \\
\hline & $800 \mathrm{~cm} 3 \sim 1200 \mathrm{~cm} 3$ & 21 \\
\hline & $<800 \mathrm{~cm} 3$ & 24 \\
\hline \multicolumn{3}{|l|}{ Breast shape } \\
\hline & Flap type & 19 \\
\hline & Hemisphere type & 18 \\
\hline & Cone type & 8 \\
\hline & pendulous type & 8 \\
\hline
\end{tabular}


Table 2. ANOVA of Comparison of Heart Dosimetry in Three Cohorts of Breast Size (small/medium/large)

\begin{tabular}{lcccc}
\hline Heart dose-volume factors & small & medium & large & $p$ \\
\hline Dmean (Gy) & $477.7 \pm 239.1$ & $658.2 \pm 263.7$ & $768.8 \pm 293.3$ & 0.01 \\
V5 (\%) & $17.5 \pm 10.4$ & $20.5 \pm 8.3$ & $24.9 \pm 9.7$ & 0.16 \\
V25 (\%) & $6.2 \pm 4.5$ & $9.2 \pm 4.8$ & $10.6 \pm 5.3$ & 0.03 \\
V30 (\%) & $5.6 \pm 3.6$ & $8.1 \pm 4.7$ & $9.7 \pm 5.0$ & 0.04 \\
V40 (\%) & $4.7 \pm 3.6$ & $6.6 \pm 4.0$ & $7.9 \pm 4.3$ & 0.09 \\
\hline
\end{tabular}

*aAbbreviation: Dmean = the mean dose of heart; V5 Gy = volume receiving at least $5 \mathrm{~Gy} ; \mathrm{V} 20 \mathrm{~Gy}=$ volume receiving at least $20 \mathrm{~Gy} ; \mathrm{V} 30 \mathrm{~Gy}=$ volume receiving at least $30 \mathrm{~Gy}$; $\mathrm{V} 40 \mathrm{~Gy}=$ volume receiving at least $40 \mathrm{~Gy}$

Table 3. ANOVA of Comparison of Heart Dosimetry in Four Kinds of Breast Shape (flap/hemisphere/cone/ pendulous)

\begin{tabular}{lccccc}
\hline Heart dose-volume factors & Flap type & Hemisphere type & Cone type & Pendulous type & $p$ \\
\hline Number & 19 & 18 & 8 & 8 & \\
Dmean (Gy) & $491.8 \pm 234.6$ & $752.7 \pm 219.0$ & $620.2 \pm 275.7$ & $666.1 \pm 238.0$ & 0.015 \\
V5 (\%) & $14.5 \pm 7.0$ & $25.1 \pm 8.3$ & $19.2 \pm 9.3$ & $24.9 \pm 11.7$ & 0.002 \\
V25 (\%) & $6.7 \pm 5.0$ & $11.1 \pm 4.8$ & $8.4 \pm 5.0$ & $8.7 \pm 5.0$ & 0.059 \\
V30 (\%) & $5.5 \pm 4.4$ & $10.1 \pm 4.6$ & $7.7 \pm 4.5$ & $7.7 \pm 3.8$ & 0.025 \\
V40 (\%) & $4.4 \pm 3.8$ & $8.2 \pm 3.9$ & $6.2 \pm 3.1$ & $6.2 \pm 3.3$ & 0.037 \\
\hline
\end{tabular}

*Abbreviation: Dmean= the mean dose of heart; V5 Gy = volume receiving at least $5 \mathrm{~Gy} ; \mathrm{V} 20 \mathrm{~Gy}=$ volume receiving at least $20 \mathrm{~Gy} ; \mathrm{V} 30 \mathrm{~Gy}=$ volume receiving at least $30 \mathrm{~Gy}$; $\mathrm{V} 40 \mathrm{~Gy}=$ volume receiving at least $40 \mathrm{~Gy}$.

Table 4. Comparison of dosimetric parameters from treatment plans obtained between the flap type and the others

\begin{tabular}{lccc}
\hline $\begin{array}{l}\text { Heart dose- } \\
\text { volume factors }\end{array}$ & $\begin{array}{c}\text { Flap } \\
\text { type }\end{array}$ & $\begin{array}{c}\text { Other } \\
\text { types }\end{array}$ & $p$ \\
\hline Dmean (Gy) & $491.8 \pm 234.6$ & $701.1 \pm 237.0$ & 0.003 \\
V5 (\%) & $14.5 \pm 7.2$ & $23.7 \pm 7.4$ & 0.001 \\
V25 (\%) & $6.7 \pm 5.0$ & $9.0 \pm 4.1$ & 0.028 \\
V30 (\%) & $5.5 \pm 4.4$ & $9.0 \pm 4.4$ & 0.008 \\
V40 (\%) & $4.4 \pm 3.8$ & $7.2 \pm 3.8$ & 0.012 \\
\hline
\end{tabular}

*Abbreviation: Dmean $=$ the mean dose of heart; V5 Gy = volume receiving at least $5 \mathrm{~Gy} ; \mathrm{V} 20 \mathrm{~Gy}=$ volume receiving at least $20 \mathrm{~Gy} ; \mathrm{V} 30$ $\mathrm{Gy}=$ volume receiving at least $30 \mathrm{~Gy} ; \mathrm{V} 40 \mathrm{~Gy}=$ volume receiving at least $40 \mathrm{~Gy}$.

Table 5. Univariate analysis of the association of MHD, DCWH with heart dose

\begin{tabular}{lll}
\hline Heart dose-volume factors & DCWH & MHD \\
\hline Dmean (Gy) & & \\
$\quad$ Pearson correlation value & -0.03 & 0.6 \\
$\quad$ Significant (two sides) & 0.013 & 0.000 \\
V25 (\%) & & \\
$\quad$ Pearson correlation value & -0.4 & 0.6 \\
$\quad$ Significant (two sides) & 0.002 & 0.000 \\
V30 (\%) & & \\
$\quad$ Pearson correlation value & -0.4 & 0.5 \\
$\quad$ Significant (two sides) & 0.003 & $<0.001$ \\
V40 (\%) & & \\
$\quad$ Pearson correlation value & -0.4 & 0.5 \\
$\quad$ Significant (two sides) & 0.004 & $<0.001$ \\
\hline
\end{tabular}

*Abbreviation: Dmean $=$ the mean dose of heart; V5 Gy $=$ volume receiving at least $5 \mathrm{~Gy} ; \mathrm{V} 20 \mathrm{~Gy}=$ volume receiving at least $20 \mathrm{~Gy} ; \mathrm{V} 30$ $\mathrm{Gy}=$ volume receiving at least $30 \mathrm{~Gy}$; V40 Gy =volume receiving at least $40 \mathrm{~Gy}$

$$
\begin{aligned}
& \mathrm{V} 25=3.6 \cdot \mathrm{MHD}+1.0(\mathrm{R} 2=0.323) ; \text { and } \\
& \mathrm{V} 25=39.3-2.3 \cdot \mathrm{DCWH}(\mathrm{R} 2=0.168) \text { respectively. }
\end{aligned}
$$

Figure 3-4 plots the heart Dmean/V25 vs. MHD/ DCWH as an example (solid dot) for all 53 left side patients. Correlations between V25 and heart mean doses vs. MHD/DCWH demonstrate difference.

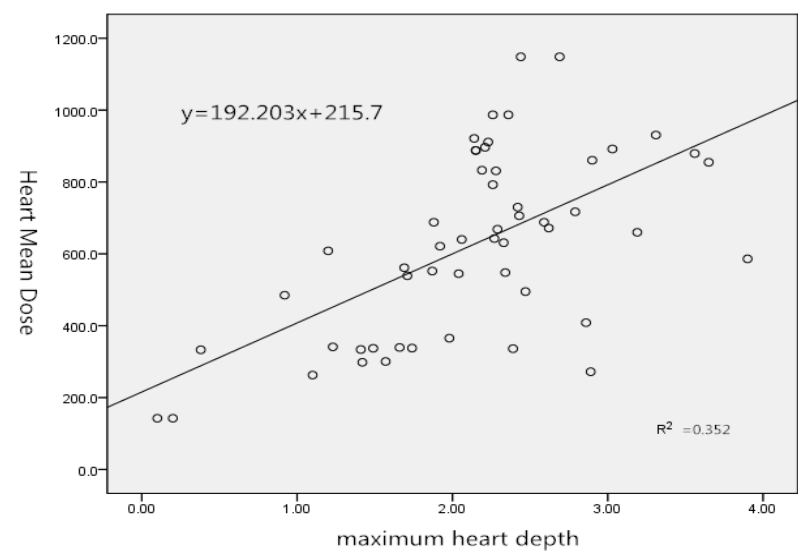

Figure 5. Scatter-plot Correlation of MHD Versus Heart Mean dose

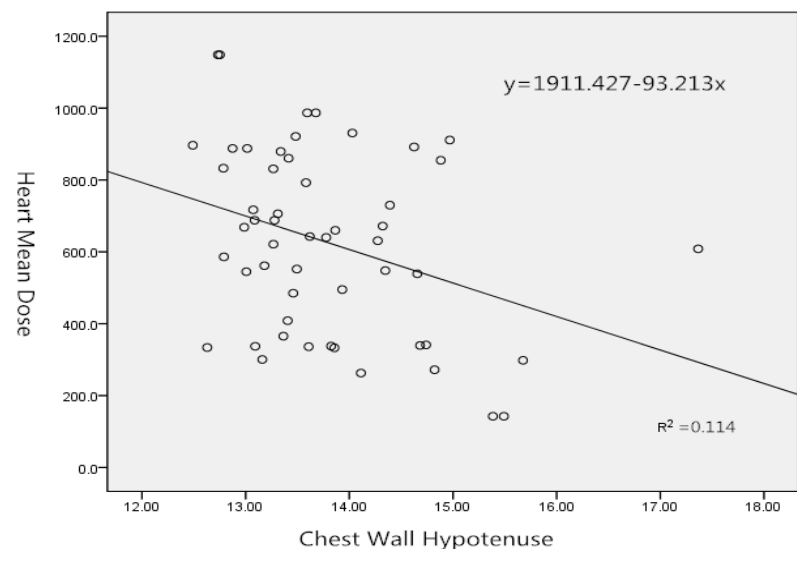

Figure 6. Scatter-plot Correlation of DCWH Versus Heart Mean dose

\section{Discussion}

Breast-conserving treatment has allowed many women to receive appropriate breast cancer management with long-term local control and survival rates equivalent to those with mastectomy, while maintaining a cosmetically acceptable breast appearance and little adverse effect 


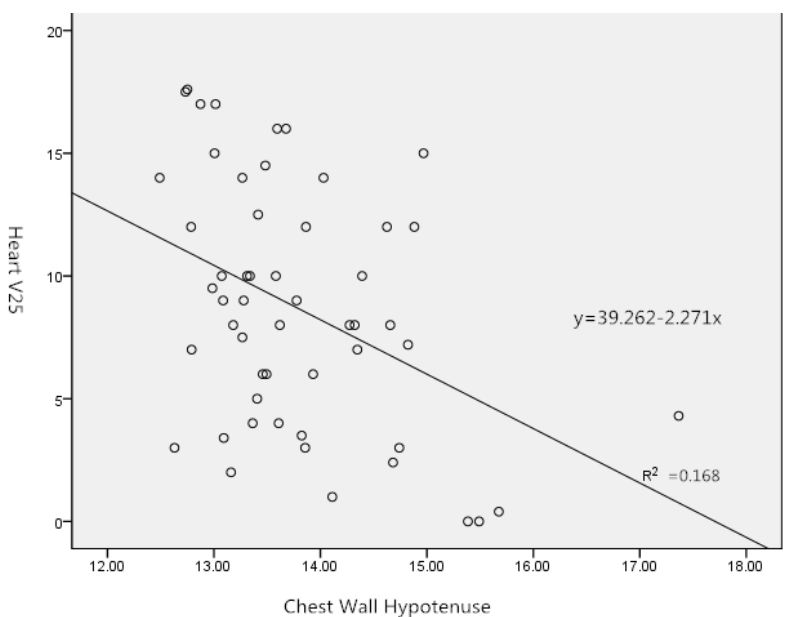

Figure 3. Scatter-plot Correlation of DCWH Versus Heart dose V25

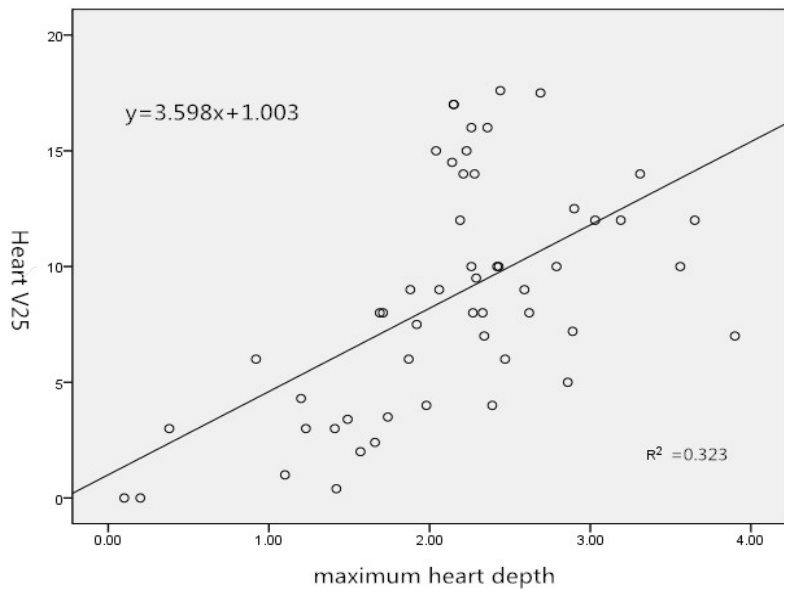

Figure 4. Scatter-plot Correlation of MHD Versus Heart dose V25

on organs at risk. All patients with breast conservation need postoperative RT. Proper RT planning is critical to ensure adequate target volume coverage and to ensure a homogeneous dose distribution (Moody et al., 1994; Clarke et al., 2005; Nandi et al., 2014).

Unfortunately, women with larger breasts and pendulous breasts can be technically challenging to treat with breast irradiation, resulting in higher rates of potential late effects, such as poor cosmesis and increased late effects (Moody et al., 1994). It is confirmed that cardiovascular mortality associated with RT for early breast cancer is correlated with the biological dose of irradiation to the heart and the irradiated volume. An excess risk of mortality from ischemic heart disease was again positively correlated with the cardiac dosimetry (Back et al., 2004). In our study, breasts were divided into different categories according to size and shape and their relationship with cardiac dose were investigated. Jointed MHD and DCWH can predict the association of dosimetric parameters with cardiac toxicity.

Here we define breast size $<800 \mathrm{~cm}^{3}$ as small breasts, breast size $>1200 \mathrm{~cm}^{3}$ as large breasts and the rest of them as medium breasts, according to generally small size of Asia women breasts. Increasing breast size leads to a small yet significant increase in dose to the heart, although the correlationship between breast size and heart dose was not statistically significant, which is consistent with prior reports (Bhatnagar et al., 2006; Hannan et al., 2012). Most studies reported that larger breasts had a correlation with dose inhomogeneity and acute skin effects, but not with cardiac dose. Our research showed no relationship between breast size and cardiac doses, which is consistent with reports concerning US and North Europe (Hannan et al., 2012). Therefore, our hypothesis was confirmed that heart dose did not correlate with breast size. Although a larger breast size requires a larger treatment field size, and therefore there is increased scatter dose to the contralateral breast. However, the IMRT allows for highly conformal plan to the breast while minimizing dose to the lung and heart, regardless of breast size. Thus, the heart dose did not correlate with the breast size in our study.

On the other hand, breast shape had a weak correlation with cardiac dose, given that we separated patients into two categories: flap type and the others. Because we found when it comes to the flap type, a more common type of breast in the Chinese, it was the most significant factor than the other three. What is interesting is that both the small size and the flap type of the breast are contributed to decrease the heart dose. Partly because the protruding length of the flap type is less than the radius of breasts base and almost all the size of flap type breasts are small. After combing the other three types, we compared them with the flap type using Student's t-test and find that there is no significant difference between two groups in heart dose but a favorable decrease trend in the flap type. From our analysis it revealed a strong statistically significant reduction in heart V5, V25, V30, V40 and mean dose in flap shape. These results can be explained if it is considered that in smaller and flap shape breasts there is a smaller beam angle and smaller chest wall separation (CWS) to make a less effect on the heart. Neal et al. (Neal et al., 1995) reported the positive correlation for breast dose inhomogeneity vs. bra cup size, breast area, and CWS. They analyzed breast size and breast dose heterogeneity for 20 patients. It is unclear whether the separation was measured at central axis or along the posterior edge in the study by Neal et al. The difference in correlation could also be attributed to the differences in definition and demarcation of PTV.

Different shapes of thoracic cage and varied delineation of the left and right border of breasts lead to different irradiation angle of tangential field, which contributes to varied CWS. But no differences were noted between the CWS and cardiac dose. In the same patient, larger CWS means higher ipsilateral irradiated lung and heart volume. In contrast, among different subsets of patients, they did not show a strong link of significant correlation. The length of thoracic cage hypotenuse reveals the shape of thoracic cage. Flat thoracic cage suggests longer thoracic cage hypotenuse with the distance between the heart and anterior chest wall more close, which leads to larger irradiated volume in WBI. Thus higher cardiac dose was caused. MHD is defined as the distance of the forefront of the heart to the chest wall hypotenuse. Similarly, larger MHD means much larger area incorporated in the tangential field, thus causing larger cardiac dose.

We can conclude that MHD/DCWH correlated with 


\section{Hui Guan et al}

heart and lung dose. These two indices can combine to make an equation, with which to access possible dose received by the patients. And this still needs a large sample of validation. We can determine whether the patient requires breath-gated techniques according to computed tomographic simulations, which helps to improve efficiency in some large medical institution.

The chest hypotenuse, which is easily acquired for treatment planning, was found to be a novel way for estimation of cardiac dose in breast treatment.

In conclusion, from our retrospective study, the shape of flap type breast showed a significant decrease of heart dose in patients with left breast cancer. While the size of breast did not show any correlationship with cardiac dose. The MDH and the DCWH were found to be the effective parameters to correlate with heart dose in tangential breast treatment. Overall, the two parameters offered a valuable and quickly reference on whether to use respiratory gating procedure before treatment planning in order to reduce the late cardiac toxicity.

With the use of IMRT in the different shape of breasts insufficiently studied, more efforts are to be made to explore the role of MHD and DCWH as parameters to access the relativity/correspondence with cardiac doses. Thus, our study contributes to the field to some extent. Although this study is limited by its retrospective nature, relatively short median follow-up (approximately 25 months), and small subgroup size, our dosimetric data and analysis remain pertinent.

DIBH with active breathing control, and respiratory gating (Beddar et al., 2007; Stranzl and Zurl, 2008; Lohr et al., 2009) have been used to reduce cardiac and pulmonary doses. Stranzl et al. (Stranzl and Zurl, 2008) reported that the irradiated cardiac volumes can be significantly reduced in left-sided breast cancer patients using DIBH technique for postoperative tangential RT. Patients with larger MHD and DCWH may particularly benefit from the gated treatment. The desired outcome for the study was to investigate a subset of breast cancer patients who are more likely to accept higher irradiation of OARs in the specific features that would be appropriate for Chinese patients and that could be determined simply and quickly in busy radiation oncology clinics.

\section{References}

Back M, Guerrieri M, Wratten C, et al (2004). Impact of radiation therapy on acute toxicity in breast conservation therapy for early breast cancer. Clin Oncol (R Coll Radiol), 16, 12-6.

Beddar AS, Kainz K, Briere TM, et al (2007). Correlation between internal fiducial tumor motion and external marker motion for liver tumors imaged with 4D-CT. Int J Radiat Oncol Biol Phys, 67, 630-8.

Bhatnagar AK, Heron DE, Deutsch M, et al (2006). Does breast size affect the scatter dose to the ipsilateral lung, heart, or contralateral breast in primary breast irradiation using intensity-modulated radiation therapy (IMRT)? Am J Clin Oncol, 29, 80-4.

Clarke M, Collins R, Darby S, et al (2005). Effects of radiotherapy and of differences in the extent of surgery for early breast cancer on local recurrence and 15-year survival: an overview of the randomised trials. Lancet, 366, 2087-106.
Darby SC, Ewertz M, McGale P, et al (2013). Risk of ischemic heart disease in women after radiotherapy for breast cancer. N Engl J Med, 368, 987-98.

Das IJ, Cheng EC, Freedman G, et al (1998). Lung and heart dose volume analyses with CT simulator in radiation treatment of breast cancer. Int J Radiat Oncol Biol Phys, 42, 11-9.

Ding Y, Li J, Wang W, et al (2014). A comparative study on the volume and localization of the internal gross target volume defined using the seroma and surgical clips based on 4DCT scan for external-beam partial breast irradiation after breast conserving surgery. Radiat Oncol, 9, 76.

Dundas KL, Atyeo J, Cox J (2007). What is a large breast? Measuring and categorizing breast size for tangential breast radiation therapy. Australas Radiol, 51, 589-93.

Group EBCTC (2000). Favourable and unfavourable effects on long-term survival of radiotherapy for early breast cancer: an overview of the randomised trials. Lancet, 355, 1757-70.

Hannan R, Thompson RF, Chen Y, et al (2012). Hypofractionated whole-breast radiation therapy: does breast size matter? Int J Radiat Oncol Biol Phys, 84, 894-901.

Lohr F, El-Haddad M, Dobler B, et al (2009). Potential effect of robust and simple IMRT approach for left-sided breast cancer on cardiac mortality. Int J Radiat Oncol Biol Phys, 74, 73-80.

Mansouri S, Naim A, Glaria L, et al (2014). Dosimetric evaluation of 3-D conformal and intensity-modulated radiotherapy for breast cancer after conservative surgery. Asian Pac J Cancer Prev, 15, 4727-32.

Moody AM, Mayles WP, Bliss JM, et al (1994). The influence of breast size on late radiation effects and association with radiotherapy dose inhomogeneity. Radiother Oncol, 33, 106-12.

Nandi M, Mahata A, Mallick I, et al (2014). Hypofractionated radiotherapy for breast cancers--preliminary results from a tertiary care center in eastern India. Asian Pac J Cancer Prev, 15, 2505-10.

Neal AJ, Torr M, Helyer S, et al (1995). Correlation of breast dose heterogeneity with breast size using 3D CT planning and dose-volume histograms. Radiother Oncol, 34, 210-8.

Qi XS, Hu A, Wang K, et al (2012). Respiration induced heart motion and indications of gated delivery for left-sided breast irradiation. Int J Radiat Oncol Biol Phys, 82, 1605-11.

Qi XS, White J, Rabinovitch R, et al (2010). Respiratory organ motion and dosimetric impact on breast and nodal irradiation. Int J Radiat Oncol Biol Phys, 78, 609-17.

Rutqvist LE, Johansson H (1990). Mortality by laterality of the primary tumour among 55,000 breast cancer patients from the Swedish Cancer Registry. Br J Cancer, 61, 866-8.

Stranzl H, Zurl B (2008). Postoperative irradiation of leftsided breast cancer patients and cardiac toxicity. does deep inspiration breath-hold (DIBH) technique protect the heart? Strahlenther Onkol, 184, 354-8.

Vicini FA, Sharpe M, Kestin L, et al (2002). Optimizing breast cancer treatment efficacy with intensity-modulated radiotherapy. Int J Radiat Oncol Biol Phys, 54, 1336-44.

Yin Y, Chen J, Sun T, et al (2012). Dosimetric research on intensity-modulated arc radiotherapy planning for left breast cancer after breast-preservation surgery. Med Dosim, 37, 287-92. 